\title{
Changes in cerebral morphology consequent to peripheral autonomic denervation
}

\author{
Hugo D. Critchley, ${ }^{\mathrm{a}, \mathrm{b}, *}$ Catriona D. Good, ${ }^{\mathrm{a}}$ John Ashburner, ${ }^{\mathrm{a}}$ Richard S. Frackowiak, ${ }^{\mathrm{a}}$ \\ Christopher J. Mathias, ${ }^{\text {b,c }}$ and Raymond J. Dolan ${ }^{\mathrm{a}}$ \\ ${ }^{a}$ Wellcome Department of Imaging Neuroscience, Institute of Neurology, UCL, 12 Queen Square, London WC1N 3BG, UK \\ ${ }^{\mathrm{b}}$ Autonomic Unit, National Hospital for Neurology \& Neurosurgery and Institute of Neurology, UCL, Queen Square, London WC1N 3BG, UK \\ ${ }^{\mathrm{c}}$ Neurovascular Medicine Unit, St. Mary's Hospital, ICSM, London W2 INY, UK
}

Received 14 June 2002; accepted 12 November 2002

\begin{abstract}
Pure autonomic failure (PAF) is characterized by an acquired, selective, peripheral denervation of the autonomic nervous system. Patients with PAF fail to generate bodily states of arousal via the autonomic nervous system in response to physical or cognitive effort. We used voxel-based morphometry to test the hypothesis that changes in the morphology of brain regions involved in autonomic control would arise as a consequence to the longstanding absence of peripheral autonomic responses in PAF patients. Optimized voxel-based morphometry of structural magnetic resonance scans was used to test for regional differences in grey and white matter in 15 PAF patients and matched controls. There were no group differences observed in global measures of grey matter, white matter, or cerebrospinal fluid (CSF). We identified morphometric differences reflecting regional decreases in grey matter volume and concentration in anterior cingulate and insular cortices in PAF patients relative to controls. Morphometric differences in brainstem and subcortical regions did not reach statistical significance. Our findings suggest that peripheral autonomic denervation is associated with grey matter loss in cortical regions encompassing areas that we have previously shown are functionally involved in generation and representation of bodily states of autonomic arousal. The nature of these changes cannot be determined from morphometric analysis alone, but we suggest that they reflect experience-dependent change consequent upon loss of afferent input to brain regions involved in representation of autonomic states.
\end{abstract}

(C) 2003 Elsevier Science (USA). All rights reserved.

Keywords: Anterior cingulate cortex; Arousal; Autonomic failure; Brain structure; Denervation; Insula; Voxel based morphometry

\section{Introduction}

Knowledge of brain mechanisms involved in control and representation of somatic states of arousal is crucial to influential theories of emotion (James, 1994; Damasio, 1994, 1999). Bodily responses mediated by the autonomic nervous system are integrated with cognitive, emotional, and physical behaviors. Studies of cerebral autonomic control in animals have focused predominantly on describing hypothalamic, brain stem, and cerebellar mechanisms of autoregulatory, homoeostatic control (Spyer, 1999) with

\footnotetext{
* Corresponding author. Wellcome Department of Imaging Neuroscience, Institute of Neurology, Queen Square, London WC1N 3BG, UK. Fax: +44-20-7813-1420.

E-mail address: h.critchley@fil.ion.ucl.ac.uk (H. Critchley).
}

less emphasis on how higher brain regions influence autonomic responses. However, across many animal species, autonomic responses can be modulated experimentally by electrical stimulation or lesions to specific neocortical, limbic, and subcortical structures (Kaada, 1951; Butcher and Cechetto, 1995). Similar findings have been observed in humans, thus direct stimulation of cingulate (Pool and Ransohoff, 1949), insula (Oppenheimer et al., 1992), and medial temporal lobe (Fish et al., 1993) can induce distinct autonomic responses, and lesions to regions including anterior cingulate, ventromedial prefrontal cortex, insula, and medial temporal lobe diminish autonomic responsiveness (Tranel and Damasio, 1994; Tranel, 2000; Tokgözoglu et al., 1999). Functional imaging studies have also implicated specific brain regions in autonomic control in humans. Thus, we have demonstrated involvement of anterior cin- 
gulate, insula, and pons in generation and representation of cardiovascular arousal (Critchley et al., 2000a), medial prefrontal cortex, and anterior insula in relation to variation in electrodermal activity (an index of sympathetic arousal) (Critchley et al., 2000b) and anterior cingulate during states of autonomic arousal during anticipation (Critchley et al., 2001a).

The central generation and rerepresentation of autonomic responses are embodied within a behaviorally integrated feedback loop. Lesions to this integrated system can provide further insight about the neuroanatomical mechanisms supporting autonomic control and pure autonomic failure (PAF) provides a unique example of a lesion to this system. Patients with PAF cannot modulate their bodily state via the autonomic nervous system but have no other neurological deficit (sensory or motor). Consequently, there is no integrated central feedback of information concerning autonomic changes that normally accompany behaviour (Mathias, 2000). PAF is characterized by a failure of neurogenic control of circulation, the cardinal feature of which is orthostatic hypotension due to an inability to activate sympathetic pathways to cause vasoconstriction during gravitational challenges (Mathias, 2000; Mathias and Bannister, 1999; Mathias et al., 1999). PAF patients do not generate increases in heart rate and blood pressure when performing effortful exercise or mental arithmetic (stressor tasks) (Mathias 1999), do not increase circulating catecholamines during physical or emotional challenge (Mathias, 1999), have absent sympathetic skin conductance responses to emotive and orienting stimuli (Magnifico et al., 1998), and have diminished pupil reflexes (Clark and Ewing, 1988). Although PAF has been associated with histological evidence of Lewy bodies in peripheral autonomic ganglia (Matthews, 1999), its ultimate cause is unknown. PAF can be distinguished from central neurodegenerative causes of autonomic failure (e.g., multiple system atrophy or Parkinson's disease with autonomic failure) by absent clinical and hormonal indicators of central neurological degeneration, and normal life expectancy (Kimber et al., 1997; Mathias, 2000; Mathias and Bannister, 1999). During effortful behaviors that normally engender autonomic arousal, functional abnormalities have been observed in PAF patients, relative to controls, including abnormalities in pons, insula, and somatosensory cortices, associated with homeostatic regulation of bodily states, and changes in cingulate activity, associated with context-dependent changes bodily arousal (Critchley et al., 2001b). During fear-conditioning, in PAF patients, the absence of peripheral arousal in response to a learnt threat-stimulus is associated with reduced activity in amygdalar and insular regions that are activated in controls (Critchley et al., 2002). These findings provide strong evidence that the cingulate, insular, pontine, and amygdalar regions are involved in autonomic control, and highlight the importance of afferent feedback in influencing central representations of autonomic responses.

Voxel-based morphometry of structural brain images provides a clinically validated means of assessing differ- ences in regional distribution of grey and white matter that may arise as a consequence of maturational and pathological processes (Good et al., 2001; Abell et al., 1999; Richardson et al., 1997; Mummery et al., 2000). Experiencedependent functional specialization may also impact upon brain morphometry. For example, increases in grey matter density have been described in taxi drivers (in the same region of posterior hippocampus that is implicated by functional neuroimaging in spatial navigation) correlated positively with years of service (Maguire et al., 2000). The present study addressed a hypothesis that chronic absence of peripheral autonomic responses would result in regional morphometric differences in specific brain areas functionally implicated in central autonomic control and representation. In this study, we apply the clinically validated, unbiased, fully automated whole brain morphometric technique to characterize and quantify the structural correlates of PAF (Good et al., 2001; Ashburner and Friston, 2000, 2001).

\section{Materials and methods}

\section{Subjects}

Fifteen subjects $(9 \mathrm{~F}, 6 \mathrm{M}$; mean age \pm SD 62 years \pm 8 ) with established diagnoses of PAF [greater than 5 years of symptoms of orthostatic hypotension and dysautonomia, without clinical evidence of nonautonomic pathology or central neurological degeneration (cerebellar signs, parkinsonism)] were recruited after full clinical assessment, together with 15 matched healthy right-handed age-matched controls (9 F, $6 \mathrm{M}$; mean age \pm SD 62 years \pm 9). Previous clinical dual-echo MRI scans for each PAF subject were examined by a neuroradiologist to exclude anyone with MRI evidence of central lesions or neurological degeneration, including syndromes such as multiple system atrophy. All subjects gave full, informed, written consent (Declaration of Helsinki, 1991) to take part in a study approved by the local Ethics Committee. Details of the PAF patients are listed in Table 1.

\section{Data acquisition}

Subjects were scanned on a 2 Tesla Siemens MAGNETOM Vision scanner. A 3D structural MRI was acquired on each subject using a T-1 weighted MPRAGE sequence (TR/TE/TI/ NEX 9.7/4/ 600/1, flip angle $12^{\circ}$, matrix size $256 \times 192$, FOV $256 \times 192$, yielding 120 sagittal slices and a slice thickness of $1.5 \mathrm{~mm}$ with in-plane resolution of $1 \mathrm{~mm} \times 1 \mathrm{~mm}$ ).

\section{Data preprocessing for voxel-based morphometry}

Data analysis was carried out with SPM99/02a (Wellcome Department of Cognitive Neurology, London, UK, http://www.fil.ion.ucl.ac.uk/spm) using Matlab5.3 (Math- 
Table 1

Pure autonomic failure patients participating in the study

\begin{tabular}{llcc}
\hline Gender & Age (years) & Duration of symptoms (years) & ADL score \\
\hline Male & 69 & 7 & 3 \\
Male & 42 & 8 & 1 \\
Male & 63 & 9 & 1 \\
Male & 55 & 11 & 9 \\
Male & 65 & 14 & 7 \\
Male & 66 & 15 & 10 \\
Female & 63 & 7 & 7 \\
Female & 61 & 8 & 1 \\
Female & 65 & 8 & 9 \\
Female & 64 & 8 & 10 \\
Female & 54 & 12 & 15 \\
Female & 61 & 13 & 10 \\
Female & 79 & 13 & 12 \\
Female & 64 & 14 & 7 \\
Female & 64 & 15 & 10
\end{tabular}

${ }^{a}$ Symptom duration was estimated retrospectively from clinical interview with patients.

${ }^{\mathrm{b}}$ ADL, activities of daily living (Katz et al., 1965), a general questionnaire scoring levels of disability in daily life. Variability in ADL score may reflect in part efficacy of treatment with hypertensive agents.

Works, Natick, MA, USA). An optimized voxel-based morphometry protocol was followed for the analysis of imaging data.

The various automated preprocessing steps implicit in VBM, namely brain extraction, spatial normalization, segmentation, modulation and smoothing have been described previously in detail (Good et al., 2001; Ashburner and Friston, 2000, 2001). Processing components pertinent to this study will be emphasized here. First, customized grey and white matter templates were created from the subjects' (patients and controls) structural MRI images. This involved spatially normalizing each structural MRI to the standard SPM T1 template, segmenting into grey and white matter and CSF, and then smoothing each grey and white matter segment with an $8 \mathrm{~mm}$ full-width at half maximum (FWHM) isotropic Gaussian kernel. Subsequently, the images were averaged to create grey and white matter templates in stereotaxic space.

The modulation step corrects volume changes introduced during the nonlinear spatial transformations. In effect, an analysis of modulated data tests for regional differences in the absolute amount (volume) of grey/white matter, whereas an analysis of unmodulated data test for regional differences in concentration of grey/white matter in the voxel. Finally, all the normalized, segmented modulated and unmodulated images were smoothed with a $12 \mathrm{~mm}$ FWHM isotropic Gaussian kernel. This conditions the residuals to conform more closely to the Gaussian random field model underlying the statistical process used for making statistically valid inferences.

\section{Statistical analysis}

Modulated and unmodulated data were analyzed separately using statistical parametric mapping (SPM99/02a) employing the framework of the General Linear Model (Friston et al., 1995b; Ashburner et al., 1997). Regionally specific differences in grey and white matter were assessed statistically, firstly, by constructing separate design matrices analysis of group differences in regional grey and white matter volume and concentration, using global (grey or white matter) signal, age, and gender of subjects as confounding covariates. Second, across the PAF patient group, we tested for morphometric changes correlating with duration of autonomic symptoms and severity of disability [scored using the Activities of Daily Living (ADL) Questionnaire (Katz et al., 1965)]. Age and gender were also included as confounding covariates in this regression analysis. Differences were reported if significance reached $P<$ 0.05 , corrected for whole brain, or when corrected for small volume of identified a priori regions of interest from human functional neuroimaging evidence, specifically published studies of autonomic control in healthy controls, and PAF patients. Family Wise Error corrections were used throughout, which approximates to a Bonferroni correction. The regions of interest were (1) anterior cingulate/medial prefrontal cortex (Critchley et al., 2000a,b, 2001a,b); (2) insulae (Critchley et al., 2000b, 2001b, 2002); (3) amygdalae (Critchley et al., 2002); and (4) pons (Critchley et al., 2000a, 2001b). Anatomical regions of interest were delineated bilaterally on an average normalized brain image (derived from all 30 subjects in the study) using MRIcro on a MSWindows platform (http://www.psychology.nottingham. ac.uk/staff/cr1/mricro.html). The average normalized brain image was the same as that used as the template within the preprocessing stage of morphometric analysis. MRIcro enables the manual delineation of volumes of interest in three planes. The anatomical boundaries of the amygdalae, anterior cingulate, and insular cortices were traced manually in three planes with reference to the anatomical atlas of Duvernoy (1991). The delineated volumes were smoothed (4 $\mathrm{mm}$ FWHM), then saved to produce mask images that reflected regional spatial extent and underlying anatomy of the a priori regions of interest and enabled simultaneous testing of contralateral areas. The bilateral anterior cingulate mask was 20392 voxels; insulae mask, 146612 voxels; amygdala mask, 1268 voxels; and the pontine mask was 7400 voxels where voxel size was $1.5 \times 1.5 \times 1.5 \mathrm{~mm}^{3}$. These masks were applied individually for significance correction (small volume correction) of regional signal differences in between-group and correlation analyses (Worsley et al., 1996).

\section{Results and discussion}

\section{Global differences in brain morphometry}

No between-group differences were observed in the comparison of PAF patients and controls in total brain volume $[\mathrm{t}(28)=0.5, P<0.05]$ concentration or in seg- 


\section{Global tissue composition}
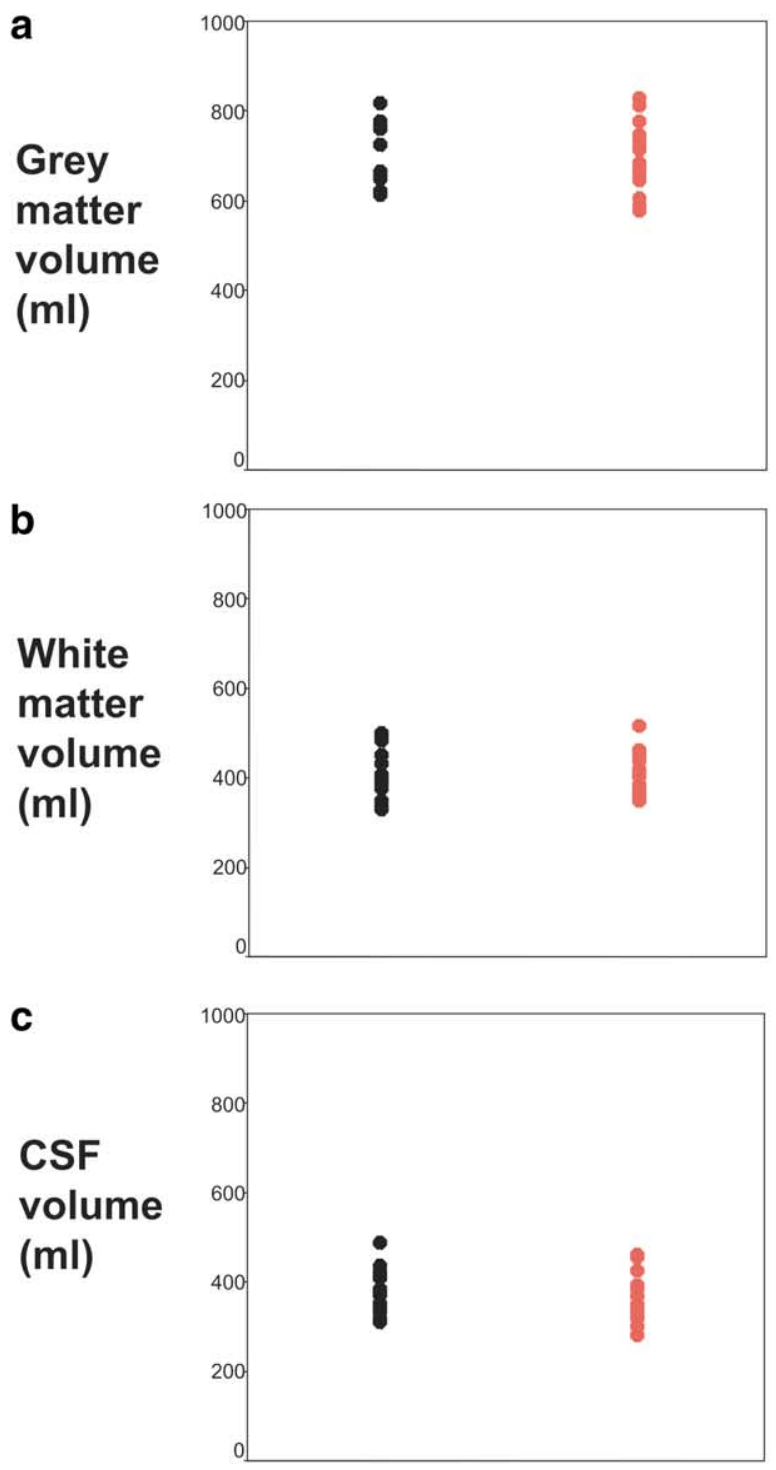

\section{Controls PAF patients}

Fig. 1. Global measures of (a) grey matter, (b) white matter, and (c) CSF volumes in controls and PAF patients. There was no significant betweengroup differences in these measures (grey matter, $\mathrm{t}(28)=0.82, P=0.4$; white matter, $\mathrm{t}(28)=0.13, P=0.9 ; \mathrm{CSF}, \mathrm{t}(28)=0.53, P=0.6)$, suggesting that there was no systematic or generalized atrophy associated with PAF.

mented volumes and concentrations of grey matter, white matter, and CSF (Fig. 1).

Regional volume and concentrations differences between $P A F$ patients and controls

PAF subjects, compared to controls, had significant reductions in grey matter volume and concentration in left anterior cingulate (peak coordinates: volume, $-11,38,27$; concentration, $-9,35,27 ; P<0.05$, corrected for bilateral anterior cingulate) (Fig. 2), and right anterior insula/frontal operculum $(48,14,10 ; 46,21,0, P<0.05$, corrected for insular mask), extending to right inferior prefrontal gyrus $(57,18,9, P=0.08)$. Reductions in grey matter concentration were observed at an uncorrected level in dorsal and ventral pons of PAF subjects, but did not reach $P<0.05$, corrected for the whole pontine region $(-17,-30,-29, P$ $=0.08$, corrected; and $3,-15,-26, P=0.12$, corrected). We observed no significant reductions in grey matter volume or concentration in amygdalae of PAF subjects (Table 2).

There were no significant regional increases in grey matter in PAF subjects relative to controls and no significant between-group differences were observed in regional volume of concentration of white matter.

\section{Within-group correlations of grey matter reductions}

We assessed whether observed morphometric differences in grey matter were influenced by the duration of autonomic symptoms or degree of disability experienced by the patients. Age and gender were treated as confounding covariates in this analysis. We could demonstrate no significant regional changes in tissue composition and morphometry directly related the duration and severity of disability across whole brain or in regions of a priori interest in regression analyses confined to the 15 PAF patients, perhaps as a result of there being few degrees of freedom. Nevertheless, the adjusted data for grey matter changes in left anterior cingulate in PAF patients, observed in the group comparison with control subjects, did negatively correlate with the ADL score of the patients (Pearson $R=-0.65, P$ $<0.05)$. This observation provides some evidence to suggest that increasing severity of the symptoms of peripheral autonomic failure is associated with increased morphological changes in an anterior cingulate region associated with autonomic control.

\section{Discussion}

Pure autonomic failure (PAF) provides a unique lesion/ deficit model for studying central control and representation of autonomic responses. Clinical investigative (physiological, biochemical, etc.) and neuropathological evidence suggests that PAF is a peripheral disorder that selectively compromises postganglionic autonomic responses (Mathias, 2000; Mathias and Bannister, 1999). In patients with PAF, we have demonstrated regional reductions in cortical grey matter in distinct brain regions implicated in generation and representation of autonomic responses. Although we only have limited evidence (confined to anterior cingulate cortex) for a relationship of these findings with severity of physical symptoms in PAF patients, we propose that this selective loss of cortical grey matter is a consequence of chronic absence of afferent feedback from the body of autonomic 
responses. The absence of a significant correlation between observed morphometric changes and illness duration does not discount this interpretation. PAF is an uncommon disorder with an insidious onset. The overlap of autonomic symptoms with a range of physical and psychological disorders may delay their recognition by patients and accurate interpretation by clinicians. Retrospective dating of symptom onset, as was conducted in this study, is also obfuscated by compensatory physiological, postural, and behavioral mechanisms in the early stages of autonomic failure. Similarly, ADL as a measure of symptom severity is also influenced by the efficacy of therapeutic interventions (Mathias, 2000; Mathias et al., 1999). These factors, coupled with the stringent analytical methods used in this VBM study, may account for insensitivity in relating regional changes in cerebral morphology to symptom duration in small sample of 15 patients.

We applied a fully automated whole brain morphometric technique to characterize the structural correlates of PAF, which avoids the idiosyncrasies of manual tracing approaches. This method has been cross-validated with functional data and region-of-interest measurements in many studies (e.g., Abell et al., 1999; Krams et al., 1999; Richardson et al., 1997; Maguire et al., 2000). Methodological details and evaluations of this technique are more fully described elsewhere (Ashburner and Friston, 2000, 2001; Good et al., 2001). Voxel-based morphometry (VBM) provides a sensitive unblased means of determining in vivo regional differences in brain tissue composition and local morphometry from MRI scans (as distinct from manual tracing of regional borders), but cannot itself provide direct information about microstructure and cytoarchitecture. In the present study we assessed regional differences in tissue volumes and grey or white matter concentration in PAF subjects relative to controls, by the addition or exclusion of the "modulation" step in the analyses (Good et al., 2001).

Although some single post mortem studies have reported Lewy bodies in brainstem of patients with the clinical diagnosis of PAF, it is important to note that there was no clinical or radiological evidence for central pathology underlying autonomic failure in the PAF patients who participated in the study. Lewy bodies in PAF are confined principally to peripheral autonomic ganglia, and PAF remains clinically and prognostically different from more pernicious conditions such as multiple system atrophy (MSA, ShyDrager syndrome) (Mathias, 2000). Also, we found no group differences in global measures of grey and white matter and CSF, consistent with normal cerebral integrity, and no generalized cerebral atrophy in PAF. Our interpretation of regional morphometric differences in PAF subjects is therefore based on a proposal that these changes arise as a direct consequence of longstanding peripheral autonomic failure.

The anterior cingulate and insula/inferior prefrontal region both showed morphometric difference in PAF patients, relative to controls. These areas have been implicated in generation and representation of autonomic states of bodily arousal. For example, functional imaging studies in healthy subjects have shown right anterior cingulate activity correlates with measures of cardiovascular arousal, such as blood pressure (Critchley et al., 2000a). Bilateral activity in anterior cingulate is modulated by the degree of anticipatory arousal (Critchley et al., 20001b), and increased anterior cingulate activity, particularly in the left hemisphere, was associated with the intention to decrease sympathetic tone during biofeedback relaxation exercises (Critchley et al., 2001c). Evidence from PAF patients also indicates that anterior cingulate contributes to generation of autonomic states of arousal. During performance of effortful tasks that normally engender cardiovascular arousal in healthy subjects, PAF patients show enhanced right anterior cingulate activity beyond that seen in controls (Critchley et al., 2001b). In these cases, as with many studies of emotional arousal states (Lane et al., 1997), dorsal regions of human anterior cingulate are implicated in autonomic regulation. Our observation of decreased grey matter concentration and volume in anterior cingulate cortex is consistent with the idea that, in PAF, a poverty of afferent information results in reduced functional representation of autonomic states and consequent morphometric adaptation. Direct afferent and efferent connections exist between anterior cingulate or insula and pontine nuclei implicated in control of central and peripheral arousal (Porrino and Goldman-Rakic, 1982), which may mediate the afferent representation of autonomic state. However, the lack of observable changes in thalamus of PAF subjects in this study or in functional imaging studies (Critchley et al., 2001a, 2002) does not exclude thalamocortical contributions to afferent transmission of arousal state to specialized visceroceptive and autonomic effector cortical areas.

Regional morphometric changes may arise as a direct consequence of changes in the rate or pattern of afferent neuronal firing. Animal studies suggest that reductions in dendritic arborization and perhaps cell loss may contribute to this involutionary process (Lenvai et al., 2000; Capurso et al., 1997). One question raised by our findings is why the left anterior cingulate showed differences in morphometry, whereas many of our functional imaging findings suggest that activity in right anterior cingulate is particularly associated with sympathetic arousal (Critchley et al., 2000a, 2001a). One possibility is that anterior cingulate activity reflects afferent modulatory influences for the control of peripheral arousal. Grey matter changes may thus arise from a lack of afferent feedback. However, in a PET study, increased right anterior cingulate activity was observed in PAF relative to control subjects during effortful tasks associated with increased sympathetic drive (Critchley, 2001b). We initially speculated that grey matter increases might be observed in right anterior cingulate consequent to this increased activity, though this was not apparent in our findings. An extension of this explanation is that left anterior cingulate may be involved in producing decreases in heart 


\section{Regional grey matter reductions in PAF}
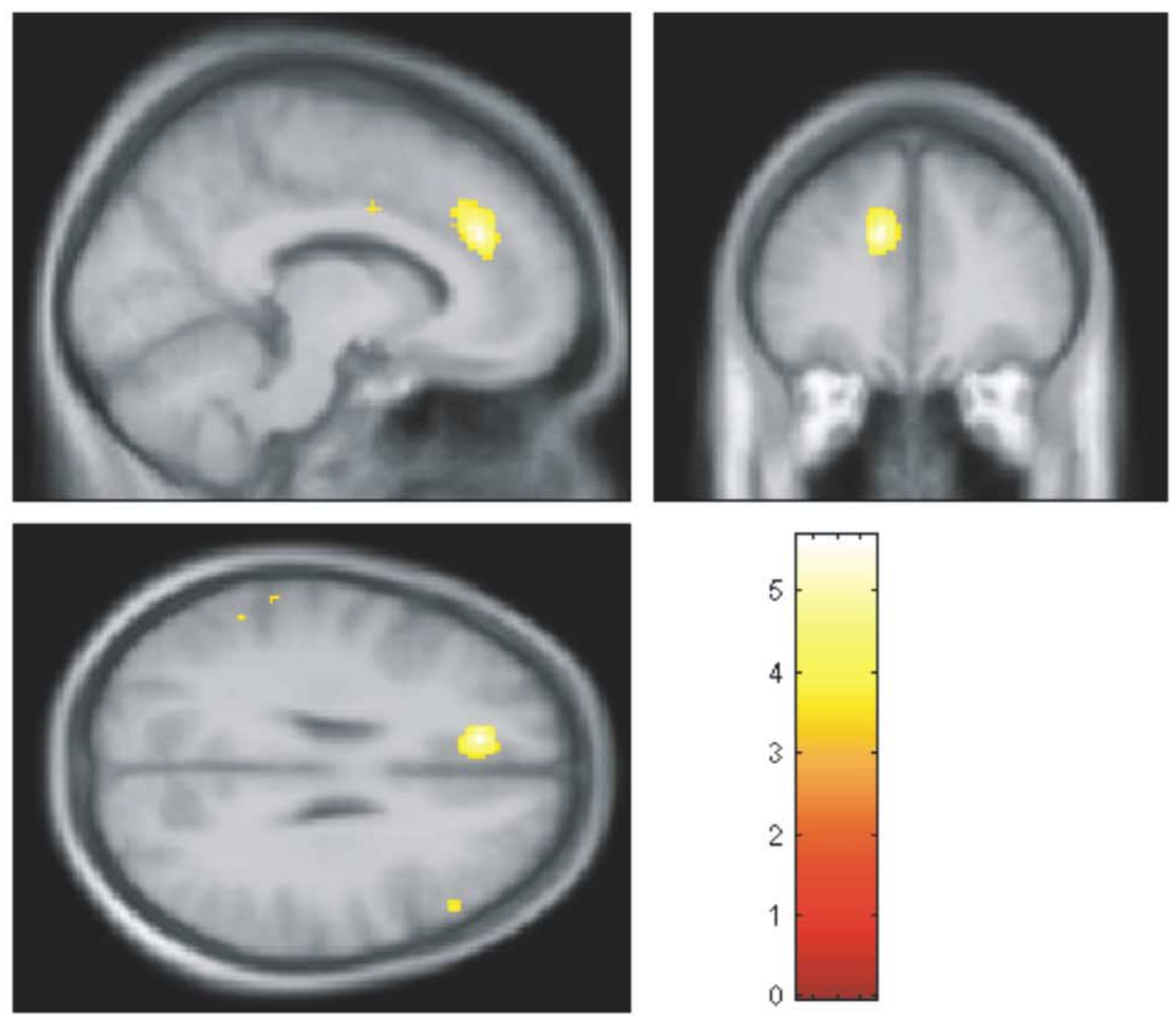

\section{Left anterior cingulate $-11,38,27$}

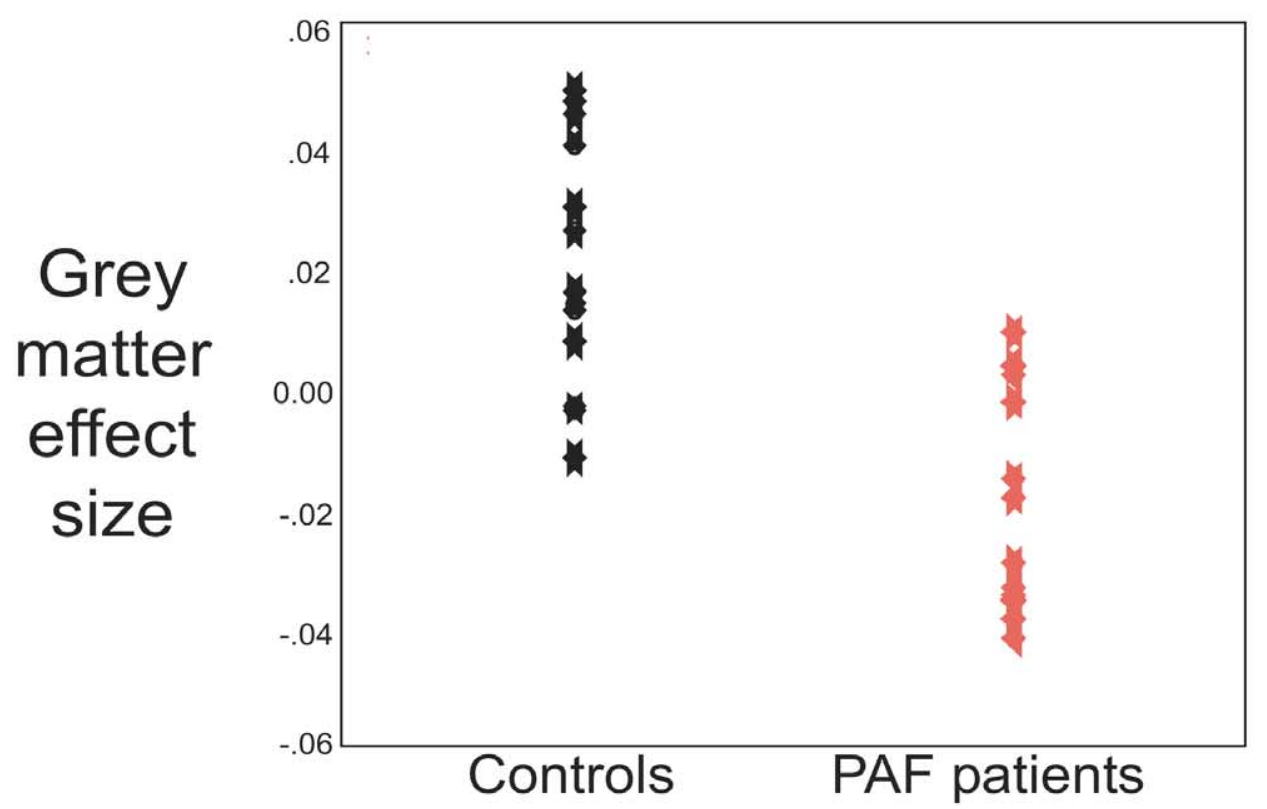

Fig. 2. Location of anterior cingulate changes in grey matter volume. Scans from each subject were transformed into normalized space and segmented into grey matter images. Each voxel subsequently modulated by the Jacobean determinants of the preceding normalization, and consequently each voxel was assigned a value reflecting the initial grey matter mass (volume). These grey matter images were then smoothed. A voxel-wide group comparison of grey matter differences between PAF subjects and controls was performed using multiple regression analyses within the general linear model. The effect size of 
Table 2

Regional grey matter changes differences between PAF patients and controls

\begin{tabular}{|c|c|c|c|c|c|c|}
\hline \multirow[t]{2}{*}{ Region } & \multicolumn{3}{|c|}{ Grey matter volume } & \multicolumn{3}{|c|}{ Grey matter concentration } \\
\hline & Side & Coordinates & $T$ value & Side & Coordinates & $T$ value \\
\hline \multirow{2}{*}{ Dorsal anterior cingulate } & $\mathrm{L}$ & $-11,38,27$ & $5.45^{\mathrm{a}}$ & $\mathrm{L}$ & $-9,35,27$ & $5.02^{\mathrm{a}}$ \\
\hline & $\mathrm{L}$ & $-9,2,36$ & 4.09 & & & \\
\hline Subgenual cingulate & - & - & - & $\mathrm{L}$ & $-6,51,-8$ & 3.95 \\
\hline \multirow[t]{2}{*}{ Anterior insula extending to inferior frontal gyrus } & $\mathrm{R}$ & $57,18,9$ & $5.46^{\mathrm{a}}$ & $\mathrm{R}$ & $54,18,6$ & 4.47 \\
\hline & $\mathrm{R}$ & $48,14,10$ & $4.19^{\mathrm{a}}$ & $\mathrm{R}$ & $48,21,0$ & $4.60^{\mathrm{a}}$ \\
\hline \multicolumn{7}{|l|}{ Pons } \\
\hline dorsal & - & - & - & $\mathrm{L}$ & $-17,-30,-29$ & 3.94 \\
\hline ventral & & & & & $3,-15,-26$ & 3.76 \\
\hline
\end{tabular}

${ }^{\text {a }}$ Significant $P<0.05$, corrected for full extent of bilateral anatomical structure.

rate and blood pressures, suggested by the role of this region in mediating volitional reduction in electrodermal activity (Critchley et al., 2001c). A system for producing decreases in heart rate and blood pressure would exacerbate symptoms of autonomic failure, and may undergo involutionary changes due to its redundancy in PAF patients. Furthermore, we did not observe grey matter differences in posterior cingulate, a region that support an evaluative representation of bodily arousal (Critchley et al., 2001b; Vogt et al., 1992). However, our findings of grey matter differences within brain areas involved in autonomic control in healthy subjects, and adjacent to regions showing functional abnormalities in PAF, share many features with previously reported observations of cortical reorganization consequent upon degraded or depleted sensory afferent input (Pons et al., 1991).

Insula cortices have an important role in the representation of somatic states and bodily responses. Consistent with this proposal, functional imaging studies report insula activity during a variety of conditions that elicit or reflect a modulation of visceral state, including micturition (Nour et al., 2000), oesophageal distension (Aziz et al., 2000), respiratory challenge (Corfield et al., 1995), thermosensory stimulation (Craig et al., 2000), cardiovascular arousal (Critchley et al., 2000a), and electrodermal activity (Critchley et al., 2000b). Functional decreases in insula activity are also seen in PAF, relative to controls (Critchley et al., 2001b). Using voxel-based morphometry, we observed decreases in grey matter in PAF within anterior insula cortex, an observation that accords with a proposed role for insula cortex in representation of bodily states of arousal. The finding again suggests involution of regional grey matter occurs in response to loss of integrated feedback of autonomic responses as a result of autonomic failure. However, this local change in tissue composition extended into prefrontal oper- culum and adjacent inferior prefrontal gyrus, regions also implicated in error detection (Menon et al., 2001). Both dorsal and subgenual regions of anterior cingulate are also implicated in emotional processing and subjective emotional experience (Damasio, 1994, 1999; Lane et al., 1997; Chua et al., 1999; Phillips et al., 1997) and in PAF patients, we have provided preliminary evidence for reduction in subjective emotional experience (Critchley et al., 2001b).

We observed only a trend for significant morphometric differences in brainstem regions, despite the expectation that differences would be observable in dorsal pons on the basis of functional imaging observations that demonstrate context-independent increases in activity of this region in PAF subjects (Critchley et al., 2001b). In this study, grey matter concentration decreases in both dorsal and ventral pons did not survive corrected significance. Brain stem regions including the pons support homoeostatic and effector mechanisms for control of autonomic responses (Spyer, 1999), and have been proposed as a site of first-order representation of bodily states (Damasio, 1999). The absence of marked morphometric differences in the pons of PAF subjects may reflect increased variability in the segmentation of pontine tissue into grey and white matter compartments. Another possibility is that increased functional activity previously observed in PAF patients in pons, and perhaps right anterior cingulate, results in a relative preservation of grey matter that maximizes residual autonomic responses during the early stages of autonomic failure. However, it is possible that morphometric adaptations to absent autonomic responses preferentially impact only on cortical brain areas involved in integrating bodily representations with behavioral experience, rather than regions involved primarily in autoregulatory processes. With this in mind, it was also of some surprise that no morphometric differences were observable in the amygdalar region. Re-

a comparison represents the regression coefficient for the adjusted measure of grey matter volume (i.e., after subtracting the mean and removing effects due to age and sex), centerd on a specified voxel in normal space. Group data indicating the location of decreases in grey matter volume associated with PAF are presented on sections of an average normalized image derived from the control and PAF subjects that took part in the study. Coronal, sagittal, and horizontal sections showing differences in left anterior cingulate, together with a color bar showing the $Z$ score of the comparison. The effect size for grey matter volume differences in the peak voxel for this contrast for each PAF and control subject is plotted beneath. 
gions within the amygdala are involved in generation of emotional autonomic responses and also mediate memory enhancement of salient material perhaps through arousal mechanisms. In PAF, neural responses within the amygdala to threat stimuli are markedly decreased in association with absent peripheral autonomic responses (Critchley et al., 2002). The explanation for why tissue morphology of some "autonomic" brain centers is affected to a greater extent than other centers remains elusive, and a subject for future study.

In summary, we examined regional brain morphometry in PAF patients who have an acquired absence of peripheral autonomic responses. Adaptive reductions in grey matter concentration and volume were observed in anterior cingulate and insula cortices, regions that have been functionally implicated in both autonomic control and also in emotional behavior. These reductions occurred in the absence of generalized changes in cerebral morphometry. Our findings extend knowledge derived from functional imaging studies about the central control of autonomic responses in humans and provides empirical evidence for adaptive changes in the adult human brain consequent to peripheral autonomic dysfunction.

\section{Acknowledgments}

This work was supported by the Wellcome Trust via Programme grants to RJD and RSF, and by a Wellcome Clinician Scientist Fellowship to HDC.

\section{References}

Abell, F., Krams, M., Ashbumer, J., Passingham, R., Friston, K., Frackowiak, R., Happe, F., Frith, C., Frith, U., 1999. The neuroanatomy of autism: a voxel-based whole brain analysis of structural scans. Neuroreport 10, 1647-1651.

Ashburner, J., Friston, K.J., 1999. Nonlinear spatial normalization using Basis functions. Hum. Brain Mapp. 7, 254-266.

Ashburner, J., Friston, K.J., 2000. Voxel-based morphometry-the methods. Neuroimage 11, 805-821.

Ashburner, J., Friston, K.J., 2001. Why voxel-based morphometry should be used. Neuroimage 14, 1238-1243.

Ashburner, J., Neelin, P., Collins, D.L., Evans, A., Friston, K., 1997. Incorporating prior knowledge into image registration. Neuroimage 6 , 344-352.

Aziz, Q., Thompson, D.G., Ng, V.W., Hamdy, S., Sarkar, S., Brammer, M.J., Bullmore, E.T., Hobson, A., Tracey, I., Gregory, L., Simmons, A., Williams, S.C., 2000. Cortical processing of human somatic and visceral sensation. J Neurosci 20, 2657-2663.

Butcher, K.S., Cechetto, D.F., 1995. Insular lesion evokes autonomic effects of stroke in normotensive and hypertensive rats. Stroke 26, $459-465$

Capurso, S.A., Calhoun, M.E., Sukhov, R.R., Mouton, P.R., Price, D.L., Koliatsos, V.E., 1997. Deafferentation causes apoptosis in cortical sensory neurons in the adult rat. J. Neurosci. 17, 7372-384.

Chua, P., Krams, M., Toni, I., Passingham, R., Dolan, R., 1999. A functional anatomy of anticipatory anxiety. Neuroimage 9, 563-571.

Clark, C.V., Ewing, D.J., 1988. Ocular autonomic function in progressive autonomic failure. Doc. Ophthalmol. 70, 309-321.
Corfield, D.R., Fink, G.R., Ramsay, S.C., Murphy, K., Harty, H.R., Watson, J.D., Adams, L., Frackowiak, R.S., Guz, A., 1995. Evidence for limbic system activation during $\mathrm{CO} 2$-stimulated breathing in man. J. Physiol. Lond. 488, 77-84.

Craig, A.D., Chen, K., Bandy, D., Reiman, E.M., 2000. Thermosensory activiation of insula cortex. Nat. Neurosci. 3, 184-190.

Critchley, H.D., Corfield, D.R., Chandler, M.P., Mathias, C.J., Dolan, R.J., 2000a. Cerebral correlates of autonomic cardiovascular arousal: a functional neuroimaging investigation. J. Physiol. Lond. 523, 259-270.

Critchley, H.D., Elliott, R., Mathias, C.J., Dolan, R.J., 2000b. Neural activity relating to the generation and representation of galvanic skin conductance response: a functional magnetic imaging study. J. Neurosci. 20, 3033-3040.

Critchley, H.D., Mathias, C.J., Dolan, R.J., 2001a. Neural activity relating to reward anticipation in the human brain. Neuron 29, 1-20.

Critchley, H.D., Mathias, C.J., Dolan, R.J., 2001b. Neural correlates of first and second-order representation of bodily states. Nat. Neurosci. 2, 207-212.

Critchley, H.D., Mathias, C.J., Dolan, R.J., 2002. Fear-conditioning in humans: the influence of awareness and arousal on functional neuroanatomy. Neuron 33, 653-663.

Critchley, H.D., Melmed, R.N., Featherstone, E., Mathias, C.J., Dolan, R.J., 2001c. Brain activity during biofeedback relaxation: a functional neuroimaging investigation. Brain 124, 1003-1012.

Damasio, A.R., 1994. Descartes' Error: Emotion, Reason and the Human Brain. Grosset/Putnam, New York.

Damasio, A.R., 1999. The Feeling of What Happens: Body and Emotion in the Making of Consciousness. Harcourt Brace, New York.

Duvernoy, H.M., 1991. The Human Brain: Surface Three Dimensional Sectional Anatomy and MRI. Springer-Verlag, New York.

Fish, D.R., Gloor, P., Quesney, F.L., Olivier, A., 1993. Clinical responses to electrical brain stimulation of the temporal and frontal lobes in patients with epilepsy. Pathophysiological implications. Brain 116, 397-414.

Friston, K., Ashburner, J., Frith, C.D., Poline, J.-B., Heather, J.D., Frackowiak, R.S.J., 1995a. Spatial registration and normalization of images. Hum. Brain Mapp. 2, 165-189.

Friston, K., Holmes, A.P., Worsley, K., Poline, J.-P., Frith, C.D., Frackowiak, R., 1995b. Statistical parametric maps in functional imaging: a general linear approach. Hum. Brain Mapp. 2, 189-210.

Good, C.D., Johnsrude, I., Ashburner, J., Henson, R.N.A., Friston, K.J., Frackowiak, R.S.J., 2001. A voxel-based morphometric study of ageing in 465 normal adult human brains. Neuroimage 14, 21-36.

James, W., 1894; 1994. Physical basis of emotion. Psychol. Rev. 1, $516-$ 529; reprinted in Psychol. Rev. 101, 205-210.

Kaada, B.R., 1951. Somato-motor, autonomic and electrocorticographic responses to electrical stimulation of rhinencephalic and other structures in primates, cat and dog. Acta Physiol. Scand. 24 (Suppl. 83), $1-285$.

Katz, S., Ford, A., Moskowitz, R., Jackson, B., Jaffe, M., 1965. Stuidies of illness in the aged; the index of ADL; a standardized measure of biological and psychosocial function. J. Am. Med. Assoc. 185, $914-$ 919.

Kimber, J.R., Watson, L., Mathias, C.J., 1997. Distinction of idiopathic Parkinson's disease from multiple-system atrophy by stimulation of growth-hormone release with clonidine. Lancet 349, 1877-1881.

Krams, M., Quinton, R., Ashburner, J., Friston, K.J., Frackowiak, R.S., Bouloux, P.M., Passingham, R.E., 1999. Kallmann's syndrome: mirror movements associated with bilateral corticospinal tract hypertrophy. Neurology 52, 816-822.

Lane, R.D., Fink, G.R., Chau, P.M., Dolan, R.J., 1997. Neural activation during selective attention to subjective emotional responses. Neuroreport 8, 3969-3972.

Lenvai, B., Stern, E.A., Chen, B., Svoboda, K., 2000. Experience-dependent plasticity of dendritic spines in the developing rat barrel cortex in vivo. Nature 404, 876-881. 
Magnifico, F., Misra, V.P., Murray, N.M., Mathias, C.J., 1998. The sympathetic skin response in peripheral autonomic failure-evaluation in pure failure pure cholinergic dysautonomia and dopamine-beta-hydroxylase deficiency. Clin. Auton. Res. 8, 133-138.

Maguire, E.A., Gadian, D.G., Johnsrude, I.S., Good, C.D., Ashburner, J., Frackowiak, R.S., Frith, C.D., 2000. Navigation-related structural change in the hippocampl of taxi drivers. Proc. Natl. Acad. Sci. USA 97, 4398-4403.

Mathias, C.J., 2000. Disorders of the autonomic nervous system. in: Bradley, W.G., Daroff, R.B., Fenichel, G.M., et al. (Eds.), Neurology in Clinical Practice. Butterworth-Heinemann, Woburn MA, pp. 2131-2165.

Mathias, C.J., Bannister, R., 1999. Investigation of autonomic disorders. in Mathias, C.J., Bannister, R. (Eds.), Autonomic Failure: A Textbook of Clinical Disorders of the Autonomic Nervous System, 4th ed. Oxford University Press, Oxford, pp. 169-195.

Mathias, C.J., Mallipeddi, R., Bleasdale-Barr, K., 1999. Symptoms associated with orthostatic hypotension in pure autonomic failure and multiple system atrophy. J. Neurol. 246, 893-898.

Matthews, M., 1999. Autonomic ganglia and preganglionic neurons in autonomic failure. in: Mathias, C.J., Bannister, R. (Eds.), Autonomic Failure: A Textbook of Clinical Disorders of the Autonomic Nervous System, 4th Ed. Oxford University Press, Oxford, pp. 169-195.

Menon, V., Adleman, N.E., White, C.D., Glover, G.H., Reiss, A.L., 2001 Error-related brain activation during a $\mathrm{Go} / \mathrm{NoGo}$ response inhibition task. Hum. Brain Mapp. 12, 131-143.

Mummery, C.J., Patterson, K., Price, C.J., Ashburner, J., Frackowiak, R.S., Hodges, J.R., 2000. A voxel-based morphometry study of semantic dementia: relationship between temporal lobe atrophy and semantic memory. Ann. Neurol. 47, 36-45.

Nour, S., Svarer, C., Kristensen, J.K., Paulson, O.B., Law, I., 2000. Cerebral activation during micturition in normal men. Brain 123, 781-789.

Oppenheimer, S.M., Gelb, A., Girvin, J.P., Hachinski, V.C., 1992. Cardiovascular effects of human insular cortex stimulation. Neurology 42, 1727-1732.

Phillips, M.L., Young, A.W., Senior, C., Brammer, M., Andrew, C., Calder, A.J., Bullmore, E.T., Perrett, D.I., Rowland, D., Williams, S.C.,
Gray, J.A., David, A.S., 1997. A specific neural substrate for perceiving facial expressions of disgust. Nature 389, 496-498.

Pons, T.P., Garraghty, P.E., Ommaya, A.K., Kaas, J.H., Taub, E., Mishkin, M., 1991. Massive cortical reorganization after sensory deafferentation in adult macaques. Science 252, 1857-1860.

Pool, J.L., Ransohoff, J., 1949. Autonomic effects on stimulating the rostral portion of the cingulate gyri in man. J. Neurophysiol. 12, 385-392.

Porrino, L.J., Goldman-Rakic, P.S., 1982. Brainstem innervation of prefrontal and annterior cingulate coyrex in the rhesus monkey revealed by retrograde transport of HRP. J. Comp. Neurol. 205, 63-76.

Richardson, M.P., Friston, K.J., Sisodiya, S.M., Koepp, M.J., Ashburner, J., Free, S.L., Brooks, D.J., Duncan, J.S., 1997. Cortical grey matter and benzodiazepine receptors in malformations of cortical development. A voxel-based comparison of structural and functional imaging data. Brain 120, 1961-1973.

Spyer, K.M., 1999. Central nervous control of the cardiovascular system. in: Mathias, C.J., Bannister, R. (Eds.), Autonomic Failure: A Textbook of Clinical Disorders of the Autonomic Nervous System, 4th Ed. Oxford University Press, Oxford, pp. 45-55.

Tokgözoglu, S.L., Batur, M.K., Topçuoglu, M.A., Saribas, O., Kes, S., Oto A., 1999. Effects of stroke localization on cardiac autonomic balance and sudden death. Stroke 30, 1307-1311.

Tranel, D., 2000. Electrodermal activity in cognitive neuroscience: neuroanatomical and neuropsychological correlates. in: Lane, R.D., Nadel, L. (Eds.), Cognitive Neuroscience of Emotion. Oxford University Press, New York, pp. 192-224.

Tranel, D., Damasio, H., 1994. Neuroanatomical correlates of electrodermal skin conductance responses. Psychophysiology 31, 427-438.

Vogt, B.A., Finch, D.M., Olson, C.R., 1992. Functional heterogeneity in cingulate cortex: the anterior executive and posterior evaluative regions. Cereb. Cortex 6, 435-443.

Worsley, K.J., Marrett, S., Neelin, P., Vandal, A.C., Friston, K., Evans, A.C., 1996. A unified statistical approach for determining significant signals in images of cerebral activation. Hum. Brain Mapp. 4, 58-73. 